ORIGINAL ARTICLE

\title{
Lipid Profile Status of Chronic Obstructive Pulmonary Disease in Hospitalized Patients
}

\author{
K Begum ${ }^{1}$, M K Begum², ZH Sarker ${ }^{3}$ \\ MRK Dewan ${ }^{4}$, MJH Siddique ${ }^{5}$ \\ ${ }^{1}$ D ept of Pathology, National Institute of D iseases of Chest and Hospital, D haka \\ ${ }^{2}$ D epartment of Pharmacy, Bangladesh U niversity, Dhaka \\ ${ }^{3}$ D epartment of Respiratory M edicine, National Institute of D iseases of Chest and Hospital, D haka \\ ${ }^{4}$ D epartment of Pathology, Dhaka M edical College, Dhaka \\ ${ }^{5}$ Department of Pathology, National Institute of Diseases of Chest and Hospital, Dhaka.
}

\begin{abstract}
The aim of this study was to evaluate the serum level of total cholesterol, triglycerides (TG), low density lipoproteins (LDL) and high density lipoproteins, (HDL) in chronic obstructive pulmonary disease (COPD) patients admitted in National Institute of Disease of Chest and Hospital, Mohakhali, Dhaka during. January 2009 to January 2010. Twenty two patients with COPD and 22 healthy controls were included in this study. Total cholesterol, HDL and TG levels were determined with ILAB 1800 Chemistry A nalyzer using ILAB test Reagents. $L D L$ concentration was calculated using the $F$ riedewald Equation. The mean level of TG was $150.04 \pm 29.66 \mathrm{mg} / \mathrm{dl}$ and $126.14 \pm 13.28$ in COPD patients and healthy control, respectively. A statistically significant difference was found between the two groups $(p<0.001)$. The mean level of TC was 181.83 \pm 20.11 $\mathrm{mg} / \mathrm{dl}$ and $176.28 \pm 15.35 \mathrm{mg} / \mathrm{dl}$ in COPD patients and healthy control respectively $(p<0.001)$. LDL level mean value was $116.12 \pm 14.26 \mathrm{mg} / \mathrm{dl}$ and $108.95 \pm 10.39$ in COPD patients and control respectively $(p<0.001)$. The mean value of HDL showed $38.79 \pm 2.4$ in COPD patients and $39.014 \pm 1.56$ in control. A statistically significance was also found between the two groups $(p<0.001)$. Our results showed that the values of TC, TG, LDL were higher than healthy control that is highly significant statistically. On the other hand, the was significantly decreased HDL level compared with controls.
\end{abstract}

Key Word: Lipid Profile, COPD Patients

\section{Introduction}

COPD is associated with significantly increased cardiovascular morbidity and mortality. A bout one million people in the UK have COPD. It mainly affects people over the age of 40 . Although primarily a lung disease, chronic obstructive airways disease (COPD) is now recognized to have important systemic consequences that may affect morbidity and mortality ${ }^{1-3}$.

Chronic obstructive pulmonary disease (COPD) is complicated by frequent exacerbations which are characterized by an increase in cough, sputum production, worsening dyspnoea, or sputum purulence 4 . Although respiratory infections are suspected to be the main factor for the exacerbations, other factors such as industrial pollutants, allergens, sedatives, congestive heart failure, and pulmonary embolism, have also been identified $4,5,6,7$. Despite the frequency and the consequences of the exacerbation of COPD (ECOPD), its underlying pathological mechanism remains poorly understood. Only a handful of studies have focused on the functional ${ }^{8}$, 
There is compelling evidence that COPD can no longer be considered a disease limited only to the lungs ${ }^{5}$. Several studies have shown that it is associated with a wide variety of systemic consequences; most notably: systemic inflammation. The mechanisms of increased cardiovascular risk in COPD remains poorly understood. CRP is increased along with a variety of acute phase proteins in patients with COPD and may contribute to the development and clinical complications of atherosclerosis in these patients. Abundant epidemiological evidence establishes the multifactorial character of this disease and indicates that ${ }^{1,2}$, underlying mechanistic link between impaired lung function ${ }^{9}$ and inflammatory $10,11,12$ aspects of the ECOPD. Nevertheless ${ }^{13}$, the effects of the multiple risk factors are at least additive. Risk factors that have been convincingly identified for arteriosclerosis of the coronary arteries are hyperlipidemia, arterial hypertension, cigarette smoking, diabetes mellitus, physical inactivity and a decrease in plasma HDL level ${ }^{14}$. The aim of this present study was to investigate the levels of total cholesterol (TCH), triglycerides (TG), low density lipoproteins (LDL), high density lipoproteins (HDL) in COPD patients.

\section{Materials and methods}

The patients with COPD without cardiac, diabetes mellitus or other systemic diseases and age and sex matched healthy controls were selected from admitted patient in National Institute of Diseases of Chest and Hospital, Mohakhali, Dhaka, during January 2009 to January 2010. Twenty-two patients (16 males, 6 females) with COPD and 22 healthy subjects ( 10 males, 12 females) were included in this study. General physical and systemic examination was carried out. The diagnosis of the patients was made according to the criteria of the A merican Thoracic Society agonist, anticholinergics and their combination, steroids and theophylline) during the study. A fter 12 hours of starvation, 5 $\mathrm{ml}$ blood samples were drawn from the subjects. The samples were centrifuged at $3500 \mathrm{rpm}$ for 10 minutes. All samples were analyzed on the same day they were taken. The lipid profile parameters such as TC, LDL, HDL and TG were assessed by using kit methods 16,17 . Total cholesterol, HDL and TG analysis were performed with ILAB 1800 Chemistry A nalyzer using IL $A B$ test reagents. Before any procedure, HDL concentration was measured with the direct method. LDL concentration was calculated using Friedewald equation formulated as: $\mathrm{LDL}$-cholesterol $=$ (total cholesterol)- $(\mathrm{HDL}$ cholesterol)-(triglyceride/5) ${ }^{18}$. All patients continued to receive their treatment. Fasting Blood sugar was tested by standard procedure. As smoking habits, only smoking status and pack of cigarettes smoked yearly at the beginning of the study were assessed; no information was collected with respect to the age of starting smoking. A nalysis of $D$ ata: $D$ ata were expressed as mean \pm standard deviation (SD) distribution of variables was tested using A NOVA test applied by $p$ value $(p<0.05)$.

\section{Results}

The mean age of the COPD patients was $55 \pm 12.52$ years and that of the controls was $53.04 \pm 12.78$ years. The age and smoking habits of our study and control groups and the results of measured parameters are shown in Table I. COPD patients showed higher serum levels of TC, TG, LDL compared to controls (Table 2). Serum concentrations of HDL were decreased and significant differences were found between the COPD patients and the controls.

Table I: Age distribution in COPD patient and Control

\begin{tabular}{llc}
\hline (years) COPD & \multicolumn{1}{c}{ Control } & \\
\hline A ge 55.09 & \pm 12.5253 .04 & \pm 12.78 \\
FS (mg/dl) 109.4 & \pm 30.89105 .166 & \pm 41.99 \\
\hline
\end{tabular}

Table II: The distribution of mean values and standard deviations of lipid parameters and Fasting sugar in COPD patients and controls

\begin{tabular}{|c|c|c|c|}
\hline $\begin{array}{l}C \\
(n==22)\end{array}$ & \multicolumn{2}{|c|}{$\begin{array}{l}\text { OPD Control } \\
\qquad(n=22)\end{array}$} & $P$ value \\
\hline $\mathrm{TG}(\mathrm{mg} / \mathrm{dL} 15$ & 50.04 & \pm 29.66126 .14 & $\pm 13.28<0.0001$ \\
\hline $\mathrm{TC}(\mathrm{mg} / \mathrm{dL})$ & 181.83 & \pm 20.11176 .28 & $\pm 15.35<0.0001$ \\
\hline $\mathrm{LDL}(\mathrm{mg} / \mathrm{dL})$ & 116.1 & $2 \pm 14.26108 .95$ & $\pm 10.39<0.0001$ \\
\hline $\mathrm{HDL}(\mathrm{mg} / \mathrm{dL})$ & 38.79 & \pm 2.439 .014 & $\pm 1.56<0.0001$ \\
\hline
\end{tabular}


Lipid Profile Status of Chronic Obstructive Pulmonary Disease

\section{Discussion}

Lipid parameter abnormalities are a highly important. Its alteration in lipid metabolism increases cardiovascular risk, and so the mortality and morbidity among diabetic patients $^{19}$, and lipoproteins metabolisms were reported in the international literature 21,20 . Quantitative and qualitative alterations in happenin22. Qualitative alterations include size difference in lipid parameters, increase in triglycerides content of $L D L$, and the susceptibility of LDL cholesterol to form peroxides, which was found specifically responsible for atherogenesis in diabetic patients ${ }^{23}$. Both lipid profile and body fat have been shown to be the important predictors for metabolic disturbances including dyslipidemia, hypertension, diabetes, cardiovascular diseases, hyperinsulinemia etc. Any alteration in the levels of lipids in body makes the individuals more prone to develop cardiovascular diseases. In this study an attempt was taken to estimate TC, TG, LDL and HLD concentrations in COPD patients. According to the results of this study, TC TG and LDL concentrations in COPD patients was significantly higher than the controls $(p=0.001)$. This result differs from the results of some former studies. Basili et al ${ }^{24}$, reported high A po B and $I p(a)$ hand, Fekete and M osler levels in female COPD patients. That the results are different might be due to differences in the characteristics of the patient groups. In the present study, their mean age was $55.09 \pm 12.52$ years. Their treatment was not interrupted because of the study. These factors may have affected the results obtained in this study. Y avuz also has shown that HDL level increases in children with asthma who use inhaled steroids 27 . The mechanism of effect was not clear. In another study, in children with asthma, the effect of slow release theophylline on lipid profile has also been reported and it was suggested that the lipid profile changes with extended use of these drugs and that this might cause an increased risk in atherosclerotic coronary heart disease ${ }^{28}$. In another study, HDL level was found to be significantly high in asthmatic patients, a finding which led to the conclusion that children with asthma and using slow release theophylline 25 found low triglyceride 29 .

Asthma and COPD are both classified as obstructive airway diseases but they differ in their pathogenesis, clinical course and treatment. The common characteristic of these diseases is that they require long-term drug use. However, the fact that these drugs change the lipid profile may increase or decrease the risk. The results obtained in the current study are in compliance with the results obtained in former studies with beta2-agonists and inhaled steroids but the fact that the patients use more than one drug simultaneously makes it harder to reach an exact decision. In this present study group, higher TG, TC, LDL and lower HDL level was found and this was unknown. These results suggest that the increases and decrease in lipid profile might be related to the drugs the patients were taking. However, since many of the patients receive a combination of drugs, a single drug cannot be held responsible for the decrease in HDL. In conclusion, there is a need for more detailed studies to understand these relationships.

\section{References}

1. van Eeden SF, Sin, DD, Respiration 2008; 75: 224-238.

2. Finkelstein J, Cha E, Scharf SM, Int. J. Chron. Obstruct. Pulmon. Dis. 2009; 4: 337-349.

3. A gusti AG. Systemic effects of chronic obstructive pulmonary disease. Proc Am Thorac Soc. 2005; 2: 367-70.

4. Global Initiative for chronic obstructive lung disease. Global Strategy for the Diagnosis, M anagement, and Prevention of Chronic Obstructive Pulmonary Disease.

5. Updated 2008; 1-19. Celli BR, MacNee W, Eur. Respir. J. 2004; 23: 932-946.

6. Tillie-L eblond I, M arquette $\mathrm{CH}$, Perez $\mathrm{T}$, Scherpereel A, Zanetti C, Tonnel AB, Remy-Jardin M, Ann. Intern. Med. 2006; 144: 390-396.

7. A kgun $M, M$ eral $M$, Onbas 0 , A raz 0 , Koplay $M$, Aslan S, M irici A, Respiration. 2006; 73: 428-433.

8. Parker CM, Voduc N, A aron SD, Webb KA, O'D onnell DE, Eur. Respir. J. 2005; 26: 420-428. 
9. Pinto-Plata VM, Livnat $G$, Girish $M, C$ abral $H$, $M$ asdin $P$, Linacre $P, D$ ew $R$, K enney $L$, Celli $B R$, Chest. 2007; 131: 37-43

10. Gompertz S, O'Brien C, Bayle DL, Hill SL, Stockley RA, Eur. Respir. J. 2001; 17: 1112-1119.

11. Groenewegen $\mathrm{KH}$, Dentener MA, Wouters $E F$, Respir. M ed. 2007; 101: 2409-2415.

12. PereraWR, Hurst JR, Wilkinson TM, Sapsford RJ, $M$ u"llerova $H$, . D onaldson GC, W edzicha JA, Eur. Respir. J. 2007; 29: 527-534.

13. Barnes PJ,. Celi BR, Eur. Respir. J. 2009; 33: 11651185.

14. Onaka L. Lipids. In: Anderson SC, Cockayne S (eds). Clinical Chemistry. Philadelphia: WB Saunders Company, 1993: 165-187

15. ATS Standards for the diagnosis and care of patients with chronic obstructive pulmonary disease. Am J Respir C rit Care M ed 1995; 153: 77-120

16. Foosati P. Serum Triglycerides determined colorimetrically with an enzyme that produces H202. Clin Chem 1982; 28: 2077-2080.

17. Richmond $W$. Preparation and Properties of a Cholesterol Oxidase from Nocardia sp. and its Application to the Enzymatic Assay of Total Cholesterol in Serum. Clin Chem 1973; 19: 1350-1356.

18. Burtis CA, A shwood ER. Lipids, Lipoproteins and A polipoproteins. Tietz Textbook of Clinical Chemistry. Third edition. Philadelphia, WB Saunders Company 1999; 843

19. Pikanen $O M, M$ artin J M, Tallman $M$, A kerblom $H$, Anderson SM. Free radical activity during developement of type 1 diabetes mellitus in rat. Life sci 1992; 50: 335-339.

20. N ucitarhan $S$, Ozben $T$, Tuncer $N$, Serum and urine malondialdehyde levels in NIDDM patients with and without hyperlipidemia. Free Radic Biol M ed 1995; 19: 893-896.
21. Libby $P, N$ athan DM, Abraham K, Brunzell JD, F ralkin JE, Haffner SM et al. Report of the national heart Lung and blood institute of Diabetes and digestive and Kidney Diseases working group on cardiovascular complications of Type 1 Diabetes M ellitus. Circulation 2005; 111: 3489-3493.

22. $Y$ oshino $G$, Hirano $T$, Kuzumi $T$, Dislipidemia in diabete mellitus. Diabetes Res Clin Pract. 1996; 1-14.

23. Kondo A, M uranaka $Y$, Ohta I, N otsa K, M anabe $M$, Kotani K, Saito K, Maekawa M, Kamo T. Relationship between triglicerides concentration and $L D L$, size evaluated by $M$ alonaldehy demodifed LDL Clin chem. 2001; 47: 893 - 900.

24. Basili S, Ferroni $P$, Vieri $M$ et al. Lipoprotein (a) serum levels in patients in affected by chronic obstructive pulmonary disease. Arteriosclerosis, 1999; 147: 249-52.

25. Fekete T, M osler R. Plasma lipoproteins in chronic obstructive pulmonary disease. Horm Metab Res 1987 Dec; 19: 661-2.

26. Floren $\mathrm{CH}, \mathrm{Kjell}$ strom $\mathrm{T}$, Bauer $\mathrm{CA}$. Bambuterol raises high-density lipoprotein levels in patients with hyperlipidaemia. J Intern M ed 1997; 42: 167-71.

27. $Y$ avuz 0, Türktafl I, Çevik $C$. The effect of high dose inhaled budesonide on lipid profile in asthmatic patients. Gen Pharmacol 1996; 27: 89-90.

28. U zuner N, Karaman O, Saydam N, Güner G. Lipoprotein profile in long term theophylline administration in children with asthma. Allergol Immunopathol (M adr) 2002 M ar-A pr; 30: 79-84.

29. Yagupsky $P$, Shahak $E, T$ al $A$, et al. Lipoprotein profile of children with asthma receiving long-term theophylline therapy: a preliminary study. J Pediatr 1992; 120: 802-5. 